\title{
The Encapsulation of Hemagglutinin in Protein Bodies Achieves a Stronger Immune Response in Mice than the Soluble Antigen
}

\author{
Anna Hofbauer ${ }^{1}$, Stanislav Melnik ${ }^{1}$, Marc Tschofen ${ }^{1}$, Elsa Arcalis ${ }^{1}$, Hoang T. Phan ${ }^{2}$, \\ Ulrike Gresch ${ }^{2}$, Johannes Lampel ${ }^{1}$, Udo Conrad ${ }^{2}$ and Eva Stoger ${ }^{1 *}$ \\ 1 Department of Applied Genetics and Cell Biology, University of Natural Resources and Life Sciences, Vienna, Austria, \\ ${ }^{2}$ Department of Molecular Genetics, Leibniz Institute of Plant Genetics and Crop Plant Research, Gatersleben, Germany
}

OPEN ACCESS

Edited by:

Domenico De Martinis,

ENEA Italian National Agency for New Technologies, Energy and Sustainable

Economic Development, Italy

Reviewed by:

Arun Kumar,

GlaxoSmithKline Vaccines, Italy Florian Krammer,

Mount Sinai Hospital, USA

${ }^{*}$ Correspondence:

Eva Stoger

eva.stoger@boku.ac.at.

Specialty section:

This article was submitted to

Plant Biotechnology,

a section of the journal

Frontiers in Plant Science

Received: 15 November 2015

Accepted: 27 January 2016

Published: 16 February 2016

Citation:

Hofbauer A, Melnik S, Tschofen M,

Arcalis E, Phan HT, Gresch U,

Lampel J, Conrad U and Stoger E

(2016) The Encapsulation

of Hemagglutinin in Protein Bodies

Achieves a Stronger Immune

Response in Mice than the Soluble

Antigen. Front. Plant Sci. 7:142.

doi: 10.3389/fpls.2016.00142
Zein is a water-insoluble polymer from maize seeds that has been widely used to produce carrier particles for the delivery of therapeutic molecules. We encapsulated a recombinant model vaccine antigen in newly formed zein bodies in planta by generating a fusion construct comprising the ectodomain of hemagglutinin subtype 5 and the $\mathrm{N}$-terminal part of $\gamma$-zein. The chimeric protein was transiently produced in tobacco leaves, and $\mathrm{H} 5$-containing protein bodies (PBs) were used to immunize mice. An immune response was achieved in all mice treated with $\mathrm{H} 5$-zein, even at low doses. The fusion to zein markedly enhanced the lgG response compared the soluble $\mathrm{H} 5$ control, and the effect was similar to a commercial adjuvant. The co-administration of adjuvants with the H5-zein bodies did not enhance the immune response any further, suggesting that the zein portion itself mediates an adjuvant effect. While the zein portion used to induce protein body formation was only weakly immunogenic, our results indicate that zein-induced PBs are promising production and delivery vehicles for subunit vaccines.

Keywords: protein bodies, molecular farming, subcellular targeting, recombinant protein, recombinant vaccine

\section{INTRODUCTION}

Polymers are widely used as carrier biomaterials for the delivery of therapeutic molecules (Petros and DeSimone, 2010). In particular, biopolymer-based nanoparticles have proven suitable for clinical applications due to their biocompatibility and biodegradability (Panyam and Labhasetwar, 2003; Nitta and Numata, 2013). A variety of materials and preparation methods have been developed for application-specific properties in terms of particle shape, surface charge, and surface features (Petros and DeSimone, 2010). Among the protein-based biopolymers, those derived from natural proteins such as silk, collagen, elastin, and fibronectin have been studied in detail (Ruszczak and Friess, 2003; Daamen et al., 2007; Lammel et al., 2010; Nitta and Numata, 2013).

Zein, a protein-based polymer found in maize seeds, has been widely used as a carrier because of favorable properties such as biocompatibility, insolubility and low water uptake, mechanical and chemical stability, and its propensity to form coatings and microparticles (Liu et al., 2005; Lai and Guo, 2011; Wang et al., 2011; Lau et al., 2013). Zein is also generally regarded as safe (GRAS) for food use and resists digestion, making it particularly suitable as an encapsulation polymer for oral drugs (Hurtado-Lopez and Murdan, 2006b; Gong et al., 2011; Lau et al., 2013; Zou and Gu, 2013; 
Ahmed et al., 2015). The intravenous delivery of drug-loaded zein-based microparticles has also been investigated as a means to achieve long-acting effects such as the slow and sustained release of pharmaceutical compounds (Lai and Guo, 2011) and more efficient drug delivery to cancer cells (Lin et al., 2011; Podaralla et al., 2012; Lohcharoenkal et al., 2014). Zein-based microspheres may also provide adjuvant effects when used as vaccine carriers (Hurtado-Lopez and Murdan, 2006a).

The in vitro loading of zein-based microparticles with drugs usually involves spray or freeze drying or liquid-liquid dispersion methods (Zhong and Jin, 2009; Podaralla and Perumal, 2010; Podaralla et al., 2012; Zou and Gu, 2013). These technical processes are expensive and can affect the activity of the encapsulated agent, e.g., the high temperatures required for spray drying are incompatible with many pharmaceutical proteins.

It is therefore appealing to use plants to achieve microencapsulation in vivo by directly incorporating recombinant proteins into naturally occurring protein storage organelles such as zein bodies (Hofbauer and Stoger, 2013). Endogenous protein storage organelles are usually found in plant seeds, and zein-like prolamins are characteristic features of cereal endosperm cells. In production systems based on cereal seeds, the recombinant protein is often targeted to accumulate in prolamin-containing storage organelles that provide a protective environment, even offering some resistance against proteolytic digestion in simulated gastric fluids (Takaiwa et al., 2015).

Instead of using the natural prolamin bodies that are formed in rice, wheat, maize or barley endosperm, it is also possible to fuse the recombinant protein to assembly sequences that induce analogous structures in tissues such as leaves, which usually lack protein storage organelles. This ectopic protein body technology bypasses the longer generation time required to produce cereal seeds while still offering the advantages of natural bioencapsulation. Sequences that share the ability to trigger the formation of ectopic protein bodies (PBs) include those derived from cereal prolamins, synthetic elastin-like peptides (ELPs) and fungal hydrophobins (Floss et al., 2008; Conley et al., 2009; Torrent et al., 2009b; Gutierrez et al., 2013; Shigemitsu et al., 2013).

One of the most widely used assembly sequences comprises the $\mathrm{N}$-terminal part of the mature $27 \mathrm{kD} \gamma$-zein protein, a member of the major prolamin-type storage protein family in maize (Shewry and Halford, 2002). Unlike other assembly sequences, it not only induces the formation of PBs but also acts as a retention sequence that stops fusion proteins from leaving the endoplasmic reticulum (ER). Consequently, the induced PBs bud from the ER as distinct round structures, underscoring the intrinsic compartment-forming properties of the zein sequence in the absence of tissue-specific factors (Mainieri et al., 2004; Llop-Tous et al., 2010). The N-terminal sequence of the $27 \mathrm{kD}$ $\gamma$-zein protein comprises two cysteine residues downstream of the signal peptide, a repeated proline-rich domain forming an amphipathic helix, and a third section that includes four additional cysteine residues (Geli et al., 1994). Several reports have confirmed that zein-derived sequences induce ectopic PBs when appended to either the $\mathrm{N}$-terminus or the C-terminus of diverse recombinant proteins, including phaseolin (Mainieri et al., 2004), enhanced cyan fluorescent protein (Llop-Tous et al., 2010), xylanase (Llop-Tous et al., 2011), DsRed (Joseph et al., 2012), and the Human papillomavirus E7 protein (Whitehead et al., 2014). Moreover, the ability to induce PBs appears to be almost entirely intrinsic and independent of other host-specific factors, thus allowing the formation of ectopic PBs in fungal, insect and mammalian cells (Torrent et al., 2009a), and the budding of PBs from ectopic membranes such as the plastid envelope, when combined with alternative subcellular targeting strategies (Hofbauer et al., 2014).

Hemagglutinin is an abundant type I integral membrane glycoprotein found on the envelope of influenza viruses and it has been widely used in influenza vaccine development and as a model antigen. The precursor protein HA0 yields two chains, i.e., HA1 $(\sim 36 \mathrm{kDa})$ and HA2 $(\sim 28 \mathrm{kDa})$, following cleavage at the motif $\mathrm{Q} / \mathrm{E}-\mathrm{X}-\mathrm{R}$. Infectivity requires both chains to be glycosylated, and also relies on the cleavage of hemagglutinin by a protease at multiple arginine residues. (Klenk and Garten, 1994; Hulse et al., 2004). The cleavage products are then covalently linked by a disulfide bond and these HA1/HA2 units form noncovalent homotrimers (Wiley et al., 1977).

A transmembrane domain is found near the C-terminus of HA2. The three-dimensional structure of hemagglutinin reveals two domains: a stem, responsible for membrane anchoring (part of HA1 and all of HA2), and a globular head (only HA1), bearing the sialic acid receptor binding domains (RBDs) (Wilson et al., 1981). In this study we generated a fusion construct comprising the ectodomain of hemagglutinin subtype 5 and the N-terminal part of $\gamma$-zein (amino acids 4-93) in order to induce the storage of the recombinant fusion protein inside newly formed PBs. The chimeric protein was transiently produced in tobacco leaves and H5-containing PBs were used to immunize mice. The resulting immune response was compared to that of control groups administered with the soluble $\mathrm{H} 5$ antigen, with or without adjuvant.

\section{MATERIALS AND METHODS}

\section{Vector Constructs}

All cloning steps were carried out using the binary vector pTRA, a derivative of pPAM (GenBank AY027531). The sequence corresponding to the H5 ectodomain (amino acids 17-520) of hemagglutinin from the A/Hatay/2004/(H5N1) influenza strain (GenBank Q5QQ29) was amplified as described (Phan et al., 2014) and a plant codon-optimized signal peptide sequence derived from a murine antibody was added to the $\mathrm{N}$-terminus to direct the protein into the secretory pathway (Vaquero et al., 1999). Amino acids 4-93 of the mature $27 \mathrm{kD}$ $\gamma$-zein protein (lacking the signal peptide) were joined to the C-terminus via a $(\mathrm{GGGS})_{2}$ linker as previously described for the phaseolin fusion construct zeolin (Mainieri et al., 2004). A $\mathrm{His}_{6}$-tag was added to the C-terminus for detection. The final expression vector "H5-Zein" was produced by transferring this coding sequence to the PTRA vector between the Tobacco etch virus (TEV) 5'-untranslated region and the Cauliflower 
mosaic virus (CaMV) 35S terminator. The expression construct was thus placed under the control of the CaMV 35S promoter with a duplicated transcriptional enhancer. An analogous construct comprising only the $\mathrm{H} 5$ ectodomain, a His 6 -tag and a C-terminal KDEL sequence was used to produce the soluble H5 antigen.

\section{Plant Material}

Tobacco (Nicotiana benthamiana) plants were cultivated in soil in a growth chamber with a 16 -h photoperiod, $26 / 16^{\circ} \mathrm{C}$ day/night temperatures and $70 \%$ relative humidity for 2 months.

\section{Agroinfiltration of Tobacco Leaves}

The expression constructs were transferred by electroporation into competent Agrobacterium tumefaciens (GV3101) cells. The bacteria were kept as a glycerol stock and used to inoculate $5-\mathrm{ml}$ aliquots of YEB medium containing $25 \mathrm{mg} / \mathrm{l}$ kanamycin, $25 \mathrm{mg} / \mathrm{l}$ rifampicin, and $50 \mathrm{mg} / \mathrm{l}$ carbenicillin. The cultures were incubated for 2 days at $28^{\circ} \mathrm{C}$, shaking at $180 \mathrm{rpm}$. Each culture was mixed 1:1 with a culture containing a silencing inhibitor (HcPro) before adjusting with $2 \mathrm{x}$ infiltration medium (100 g/l sucrose, $3.6 \mathrm{~g} / \mathrm{l}$ glucose, $8.6 \mathrm{~g} / \mathrm{l} \mathrm{MS}$ salts, $\mathrm{pH}$ 5.6) to an $\mathrm{OD}_{600}$ of $\sim 1.0$. After adding $200 \mu \mathrm{M}$ acetosyringone, $N$. benthamiana leaves were infiltrated using a syringe (for smallscale expression) or vacuum (for large-scale expression). Young plants were completely submerged in the suspension and vacuum was applied for $2 \mathrm{~min}$. The infiltrated leaves were harvested 7 days post-infiltration (DPI).

\section{Protein Purification}

Soluble H5: Immobilized Metal Affinity Chromatography (IMAC)

Frozen leaf powder was mixed at a ratio of $1: 2(\mathrm{w} / \mathrm{w})$ with cold lysis buffer (50 mM sodium phosphate buffer, pH 8.0, $300 \mathrm{mM}$ $\mathrm{NaCl}, 5 \mathrm{mM}$ imidazole, $0.5 \mathrm{mM}$ PMSF) and sonicated briefly to induce further cell lysis. After $2 \mathrm{~h}$, the suspension was centrifuged at $9000 \mathrm{rpm}$ for $20 \mathrm{~min}$ and the supernatant was passed through a $1-\mu \mathrm{m}$ filter. The $\mathrm{pH}$ was re-adjusted to 8.0 and the suspension was centrifuged as above. The supernatant was passed through a $0.45-\mu \mathrm{m}$ filter before mixing with Ni-IDA IMAC resin (BioRad, Munich, Germany). Approximately $2 \mathrm{ml}$ of $50 \%$ resin suspension was added per $50 \mathrm{ml}$ supernatant. After incubation for $1 \mathrm{~h}$, the resin was loaded onto a column and washed with eight volumes of wash buffer ( $50 \mathrm{mM}$ sodium phosphate buffer, $\mathrm{pH} 8.0,300 \mathrm{mM}$ $\mathrm{NaCl}, 10 \mathrm{mM}$ imidazole). The protein was eluted with elution buffer (50 mM sodium phosphate buffer, pH 8.0, $300 \mathrm{mM} \mathrm{NaCl}$, $250 \mathrm{mM}$ imidazole). The amount of protein in each fraction was determined using the Bradford assay before immunoblot analysis.

\section{Protein Bodies (Density Gradient Centrifugation)}

The frozen leaf powder was mixed 1:1 (w/v) with extraction buffer (10 mM Tris- $\mathrm{HCl}, 0.4 \mathrm{M}$ sucrose, $\mathrm{pH}$ 7.5) and incubated overnight at $4^{\circ} \mathrm{C}$ with constant shaking. The homogenate was passed through two layers of miracloth to remove solid debris and then loaded on a discontinuous sucrose gradient $(3,2.5,2$, 1.5 , and $1 \mathrm{M}$ in $10 \mathrm{mM}$ Tris- $\mathrm{HCl}, \mathrm{pH}$ 7.5). This preparation was centrifuged at $30,000 \mathrm{rpm}$ and $4^{\circ} \mathrm{C}$ for $3 \mathrm{~h}$ in a Beckman ultracentrifuge (SW 41Ti or SW32TI rotor). After separation, $500-\mu 1$ fractions were collected for analysis by SDS-PAGE and immunoblotting.

\section{Protein PAGE and Immunoblot Analysis}

Infiltrated leaves (7 DPI) were harvested and ground in liquid nitrogen to a fine powder. We then extracted $60 \mathrm{mg}$ of leaf powder in $200 \mu \mathrm{l}$ buffer $\mathrm{K}(62.5 \mathrm{mM}$ Tris, $\mathrm{pH} 7.4,10 \%$ glycine, 5\% 2-mercaptoethanol, 2\% SDS, $8 \mathrm{M}$ urea). Ten microliter of the extract were mixed with loading buffer and boiled for $10 \mathrm{~min}$ before loading. Samples collected from the density gradient and IMAC procedures were mixed with $5 \mathrm{x}$ loading buffer, boiled at $100^{\circ} \mathrm{C}$ for $10 \mathrm{~min}$ and separated by reducing SDS-PAGE (12\% polyacrylamide gel, $200 \mathrm{~V}$ for $90 \mathrm{~min}$ ). Gels were stained with Coomassie Blue or transferred to a nitrocellulose membrane. The membrane was blocked with $5 \%(\mathrm{w} / \mathrm{v})$ skimmed milk in phosphate buffered saline (PBS) for $1 \mathrm{~h}$ and then incubated with a mouse anti-poly-histidine antibody (Sigma-Aldrich Chemie GmbH, Germany) at room temperature for $2 \mathrm{~h}$, diluted 1:10000. The blot was washed three times in PBS plus 0.05\% Tween-20 (PBST) and then incubated for $1 \mathrm{~h}$ with the secondary anti-mouse alkaline phosphataseconjugated antibody (diluted 1:5000). The membrane was washed another three times with PBST and the signal was detected using the NBT/BCIP system. For quantitation, the samples were compared to serial dilutions of a $\mathrm{His}_{6}$-tagged standard protein, and the images were analyzed using BioRad Image Lab v5.1.

\section{Fluorescence Microscopy}

Infiltrated leaves were cut into small pieces with a razor blade and fixed in $4 \%(\mathrm{w} / \mathrm{v})$ paraformaldehyde plus $0.5 \%(\mathrm{v} / \mathrm{v})$ glutaraldehyde in $0.1 \mathrm{M}$ phosphate buffer $(\mathrm{pH} 7.4)$ at $4^{\circ} \mathrm{C}$ overnight. For immunolocalization by confocal microscopy, vibratome sections were mounted on a glass slide, blocked with $5 \%(\mathrm{w} / \mathrm{v})$ bovine serum albumin (BSA) in $0.1 \mathrm{M}$ phosphate buffer $(\mathrm{pH}$ 7.4) and incubated with a polyclonal antibody against $27 \mathrm{kD} \quad \gamma$-zein. The samples were then incubated with an AlexaFluor ${ }^{\circledR} 488$-conjugated secondary antibody and observed under a Leica SP5 confocal laser scanning microscope (CLSM).

\section{Immunization of Mice}

Male BL6 C57/Bacl6J mice (6-8 weeks old) obtained from Charles River Laboratories, Research Models and Services were assigned to seven groups $(n=10)$. Immunization was carried out by the subcutaneous injection of 150 or $300 \mathrm{ng}$ of H5-zein either with or without Freund's adjuvant (1:1) (Difco Laboratories, Detroit, Michigan). For the primary immunization, complete Freund's adjuvant was used where indicated. Booster immunizations consisted of two additional injections of the same antigen (with or without incomplete Freund's adjuvant). As controls, one group received PBS with Freund's adjuvant only, and two groups were injected three times with soluble H5 (15 $\mu \mathrm{g})$, with or without adjuvant. After the third immunization, the mice were retro-orbitally 
bled and serum samples were collected for individual testing. A second set of blood samples was taken 8 weeks after the primary immunization and the mice were sacrificed immediately afterward.

The animal experiments were approved by the Landesverwaltungsamt Sachsen-Anhalt, Halle/Saale, Referat Verbraucherschutz, Veterinärangelegenheiten and by the Landkreis Harz, Amt für Veterinärwesen und Lebensmittelüberwachung, Halberstadt. All animals received humane care according to the requirements of the German Animal Welfare Act, $\$ 8$ Abs. 1 .

\section{IgG Quantitation by ELISA}

The wells of a flat-bottom microtiter plate were coated with $0.2 \mu \mathrm{g}$ per well of the antigen (purified recombinant $\mathrm{H} 5$ or zein (Sigma-Aldrich Chemie GmbH, Germany)). Then $100 \mu l$ of diluted serum (1:250 in PBS with 3\% BSA) was added to each well and incubated at room temperature for $1.5 \mathrm{~h}$. Rabbit anti-mouse IgG alkaline phosphatase-conjugated antibody (diluted 1:2000 in PBST) was used for detection. The IgG titer was determined by adding immune serum samples as serial dilutions starting at 1:1000. Curve fitting by five-parameter logistic regression was used to calculate the endpoint titer for each mouse. End-point titers were determined as the reciprocal highest serum dilutions that produced mean optical density values two-fold greater than the geometric mean of those from the negative control (injected with PBS) sera. The statistical significance was determined using Student's $t$-test $\left({ }^{* *} p<0.01 ;{ }^{*} p<0.1\right)$.

Selected ELISA experiments were carried out using recombinant $\mathrm{H} 5$ purified via an additional size exclusion chromatography step. The same results were obtained, indicating that the H5 preparation purified via IMAC was sufficiently pure for coating, and did not contain significant amounts of immunoreactive impurities.

\section{Hemagglutination Inhibition (HI) Test}

HI tests were carried out as described by Phan et al. (2013). Briefly, a $25-\mu \mathrm{l}$ aliquot of murine serum was mixed with $25 \mu \mathrm{l}$ PBS and added to the first well of a V-bottom microtiter plate. Twofold serial dilutions were prepared across the row of 12 wells. Aliquots $(25 \mu \mathrm{l})$ containing $4 \mathrm{HAU}$ of inactivated virus [A/swan/Germany/R65/2006(H5N1)] were added to each well and incubated for $30 \mathrm{~min}$ at room temperature. We then pipetted $25 \mu \mathrm{l}$ of a $1 \%$ red blood cells (RBCs) suspension into each well and the plate was again incubated for $30 \mathrm{~min}$ at room temperature. The HI titer was defined as the reciprocal of the highest serum dilution that achieved the complete inhibition of hemagglutination.

\section{RESULTS}

\section{Hemagglutinin-Zein Fusions form PBs in $N$. benthamiana Leaves}

Expression vector "H5-Zein" containing the sequence corresponding to the $\mathrm{H} 5$ ectodomain of hemagglutinin,

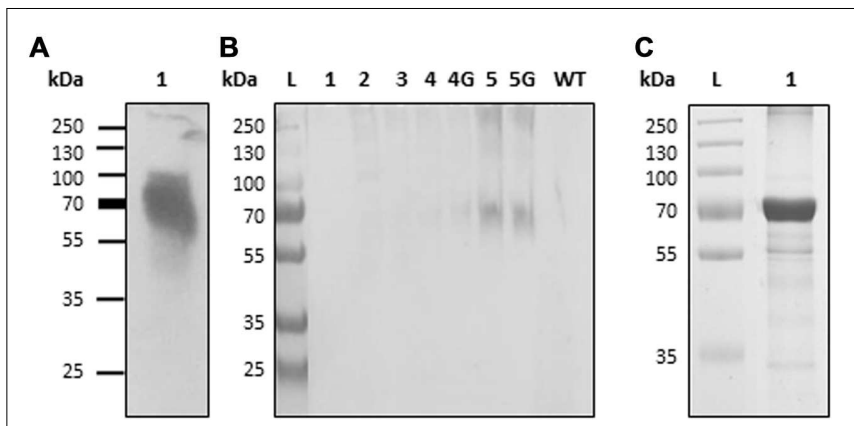

FIGURE 1 | Production and isolation of H5-zein and H5. (A) Immunoblot analysis of a leaf extract containing $\mathrm{H} 5$-zein. (B) Immunoblot of $\mathrm{H} 5$-zein samples following the sucrose density gradient separation of leaf homogenates. Lanes 1-5: Selected samples from different density steps along the gradient are shown (lane 1: sample from the top of the gradient; lane 5: pellet. $4 \mathrm{G}$ and $5 \mathrm{G}$ are the same fractions as 4 and 5, but obtained from a different experiment). An antibody specific for the His 6 tag was used for detection. (C) H5 after IMAC purification (3 $\mu \mathrm{g}$ were leaded on the gel and stained with Coomassie). wt, wild type; $L$, molecular weight ladder in kDa.

fused to amino acids 4-93 of the mature $27 \mathrm{kD} \gamma$-zein protein, was introduced into $N$. benthamiana leaves by agroinfiltration. Immunoblot analysis of extracts from the infiltrated leaves 7 DPI revealed the presence of a band corresponding to the fusion protein (Figure 1A). The higher than predicted molecular mass probably reflected the glycosylation of $\mathrm{H} 5$, as previously reported (Phan et al., 2014). The fusion of H5 to zein resulted in the formation of $\mathrm{PBs}$, whose presence was confirmed by immunofluorescence microscopy. All labeling was concentrated in the PBs, whereas no signal was detected in the ER lumen or in the apoplast (Figure 2). This result was confirmed by the density step gradient centrifugation of leaf homogenates (Figure 1B). The gradient fractions were collected and tested by immunoblot analysis. No recombinant fusion protein was found in fractions from the top of the gradient, a small amount was present in the highest density fractions, and the majority of the fusion protein was found in the pellet (Figure 1B), similar to results reported with another zein fusion protein that forms PBs (Whitehead et al., 2014). Sucrose was removed by pooling the selected fractions and resuspending them in $10 \mathrm{mM}$ Tris ( $\mathrm{pH}$ 7.5) before centrifuging them under the same conditions as above. The supernatant was removed and the pellet was re-suspended in sterile PBS. The protein suspension was stored at $-20^{\circ} \mathrm{C}$ prior to the immunization experiments. A total of $\sim 120 \mu \mathrm{g}$ H5-zein was recovered from $300 \mathrm{~g}$ of fresh infiltrated leaves. Soluble H5 lacking the zein fusion was expressed as a control and purified by IMAC as previously described (Phan et al., 2013). We recovered $1 \mathrm{mg}$ of $\mathrm{H} 5$ from $500 \mathrm{~g}$ of fresh infiltrated leaves, and this was used as a positive control for immunization (Figure 1C).

\section{H5-Zein PBs Elicit an Immune Response in Mice}

The H5-zein protein body suspension and the soluble $\mathrm{H} 5$ antigen were each used to immunize mice (Figure 3). The 


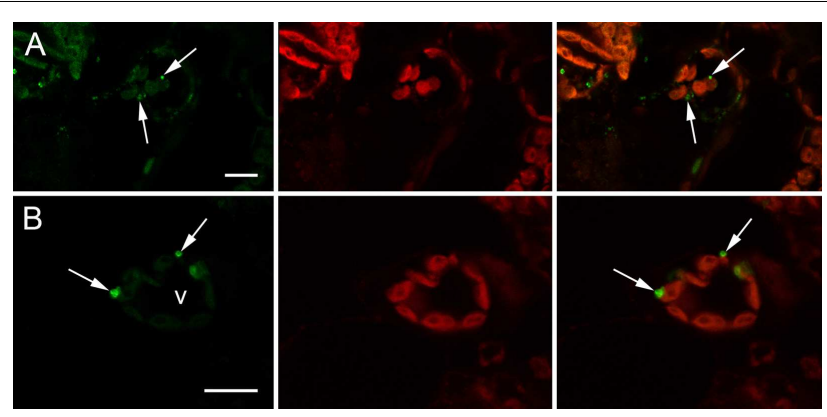

FIGURE 2 | Immunolocalization of H5-zein in $\mathbf{N}$. benthamiana leaves by confocal microscopy. (A) Abundant H5-zein protein bodies (PBs) can be observed in mesophyll cells (arrows). (B) H5-zein PBs are 1-2 $\mu \mathrm{m}$ in diameter and can be found in the cytoplasm between the chloroplasts (arrows). The left panel shows the detection of the fusion protein. The middle panel shows autofluorescence of the chloroplasts. The right panel shows the overlay pictures. Abbreviations: v, vacuole. Bars $=10 \mu \mathrm{m}$.

mice were allocated to seven groups ( $n=10$ per group) and immunization was carried out by the subcutaneous injection of 150 or $300 \mathrm{ng}$ of H5-zein either with or without Freund's adjuvant. This low dosage of H5-zein bodies was chosen to confirm the hypothesis that particulate antigens are effective in small amounts. As controls, two groups were injected three times with soluble H5 (15 $\mu \mathrm{g}$, a dose previously confirmed to provoke a strong humoral immune response), one with and one without adjuvant.

The plant-derived H5-zein protein body suspension was shown to elicit an IgG response in $100 \%$ of the animals, even at low doses. Interestingly, the addition of an adjuvant to the H5-zein bodies did not cause a significantly stronger immune response (Figure 3, groups 1 vs. 3 and 2 vs. 4), whereas the adjuvant had a significant impact in the control groups receiving soluble H5 (Figure 3, groups 5 vs. 6).

\section{The Zein Fusion Component is Only Weakly Immunogenic but has a Significant Adjuvant Activity}

To confirm the observations summarized above, we carried out an in-depth comparison of IgG titers of groups 3, 5, and 6 . The IgG response elicited by $\mathrm{H} 5$-zein without adjuvant (group 3) was comparable to that achieved by injecting soluble $\mathrm{H} 5$ combined with an adjuvant (group 5). In contrast, the administration of soluble $\mathrm{H} 5$ without adjuvant elicited a minimal IgG response (Figure 4A). This suggested that the zein component and/or the particulate nature of the protein body act as an adjuvant.

To determine whether the zein portion fused to $\mathrm{H} 5$ has intrinsic immunogenic properties, we investigated the IgG response directed against zein by comparing the IgG response in groups 1 (H5-zein with adjuvant), 3 (H5-zein without

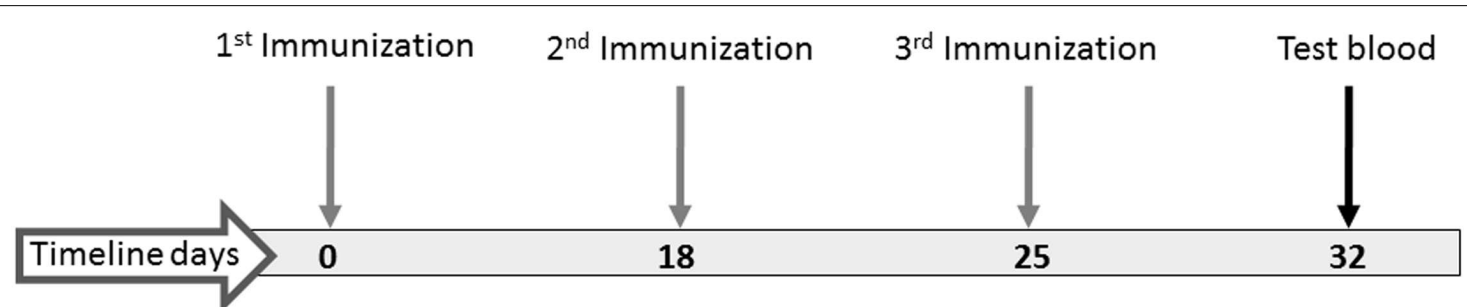

A

\begin{tabular}{|l|l|l|}
\hline Group & Immunogen & $\begin{array}{l}\text { Dosage/ } \\
\text { Adjuvant }\end{array}$ \\
\hline $\mathbf{1}$ & H5-zein & $50 \mu \mathrm{l}+$ adjuvant \\
\hline $\mathbf{2}$ & H5-zein & $25 \mu \mathrm{l}+$ adjuvant \\
\hline $\mathbf{3}$ & H5-zein & $50 \mu \mathrm{l}$ \\
\hline $\mathbf{4}$ & H5-zein & $25 \mu \mathrm{l}$ \\
\hline $\mathbf{5}$ & H5 & $25 \mu \mathrm{l}$ +adjuvant \\
\hline $\mathbf{6}$ & H5 & $25 \mu \mathrm{l}$ \\
\hline $\mathbf{7}$ & PBS & $25 \mu \mathrm{l}+$ adjuvant \\
\hline
\end{tabular}

B

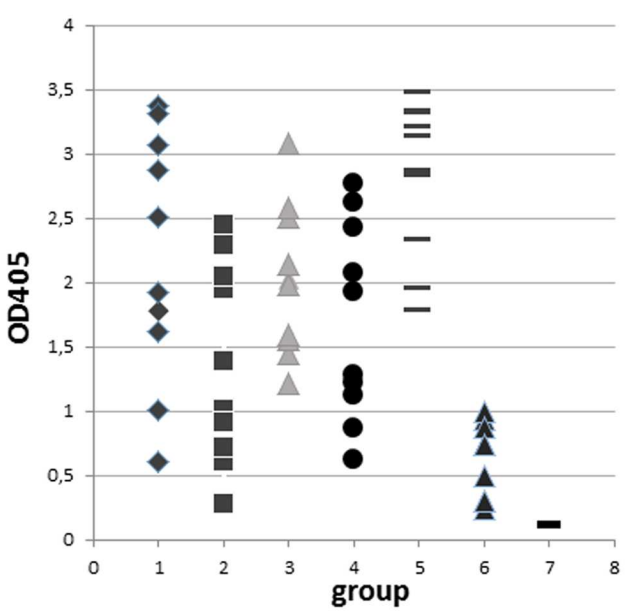

FIGURE 3 | Immunization timeline and IgG responses in the seven treatment groups. (A) All mice were injected with a primary dose of H5-zein (6 $\mu \mathrm{g} / \mathrm{ml})$ or H5 $(0.6 \mathrm{mg} / \mathrm{ml})$, both in sterile PBS, with or without Freund's complete adjuvant. For the second and third injections, the adjuvant was switched to Freund's incomplete adjuvant. (B) ELISA analysis of the anti-H5 IgG response following the third immunization. A single dot represents the ELISA result from a single serum sample. Each treatment group comprised 10 mice. 
A

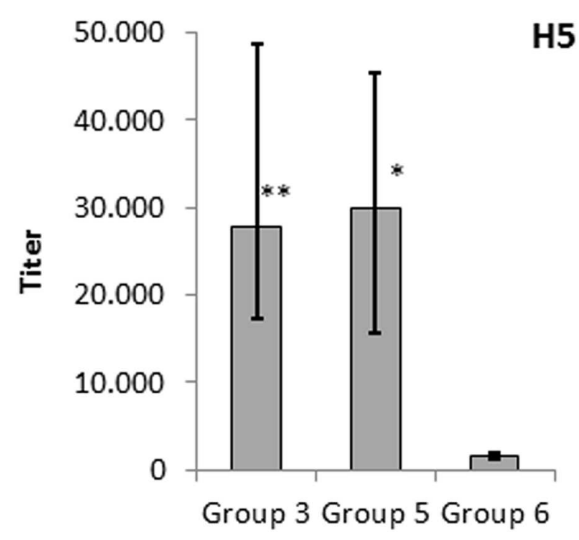

B

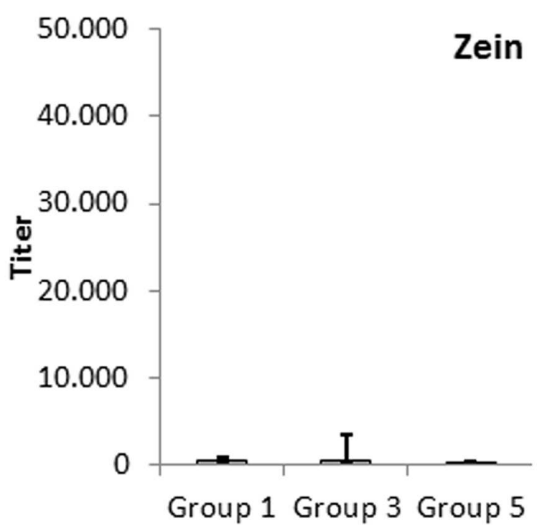

FIGURE 4 | Serum IgG response against (A) H5 and (B) zein. Data represent the median endpoint titer per group, whereas the lower and upper ends of the error bars represent the first and third quartiles, respectively. (A) Statistical analysis to compare group 3 (H5-zein without adjuvant) and group 5 (H5 with adjuvant) vs. group 6 (H5 without adjuvant). (B) Statistical analysis to compare group 1 (H5-zein with adjuvant) and group 3 (H5-zein without adjuvant) vs. group 5 (H5 with adjuvant). Each treatment group comprised 10 mice. Statistical significance was determined by Student's $t$-test $\left(* * p<0.01\right.$; ${ }^{*} p<0.1$; no asterisk indicates $p>0.1)$.

adjuvant), and 5 (soluble $\mathrm{H} 5$ with adjuvant). Although we generally detected low IgG titers, $30 \%$ of the animals in groups 1 and 3 showed a clearly detectable IgG response against zein (Figure 4B). Overall the immune response in the treatment groups was not significantly different to that of the control group $(p>0.1)$.

\section{HI Antibody Titers are Insignificant}

All mice vaccinated with $\mathrm{H} 5$-zein $\mathrm{PBs}$ showed an immunological response so we carried out $\mathrm{HI}$ tests on the serum from each mouse to determine whether the induced antibodies were potentially capable of neutralizing the virus. Because of the unavailability of the A/Hatay/2004(H5N1) virus in an inactivated form, the heterologous inactivated virus strain A/swan/Germany/R65/2006(H5N1) was used for the HI assay. The deduced hemagglutinin amino acid sequence similarity of both strains is 96\%, and it was previously shown that HI titres against inactivated virus A/swan/Germany/R65/2006 (H5N1) could be measured in sera from mice vaccinated with trimeric $\mathrm{HA}$ derived from the $\mathrm{HA}$ sequence corresponding to the A/Hatay/2004(H5N1) virus (Phan et al., 2013). The HI assay results indicated that the $\mathrm{HI}$ antibody titers were either below or marginally above the detection limit in all treatment groups (Table 1).

TABLE 1 | $\mathrm{HI}$ titers against inactivated virus

[A/swan/Germany/R65/2006(H5N1)].

\begin{tabular}{lc}
\hline Treatment group & Geometric mean titer \\
\hline 1 (H5-zein + adjuvant) & 6,06 \\
3 (H5-zein) & 4,29 \\
6 (H5) & 3,4 \\
7 (PBS) & 7,46
\end{tabular}

\section{DISCUSSION}

The expression of recombinant proteins in plants is an attractive strategy reflecting the versatility, safety, scalability, and economy of plant-based production platforms (Rybicki et al., 2013; Stoger et al., 2014). Plants also offer the possibility to accumulate recombinant pharmaceutical proteins within endogenous or ectopic protein storage organelles, which can either be derived directly from the ER or represent protein storage vacuoles (Khan et al., 2012). Here, we successfully induced the formation of ectopic $\mathrm{PBs}$ by fusing the $\mathrm{H} 5$ ectodomain of hemagglutinin to the $\mathrm{N}$-terminal sequence of $\gamma$-zein. Previous studies have shown that the biogenesis of PBs by zein is influenced by the fusion partner, and that not all fusion proteins support the efficient formation of PBs. For example, phaseolin induces the efficient formation of zeolin PBs when fused to the N-terminal sequence of $\gamma$-zein (Mainieri et al., 2004). However, PBs were not formed when the Human immunodeficiency virus Nef antigen was fused to the same $\gamma$-zein sequence, but protein body formation was possible again when Nef was fused to the entire chimeric protein zeolin (de Virgilio et al., 2008).

The induction of H5-containing protein aggregates in plants has also been achieved by fusing the antigen to hydrophobin and ELPs (Phan et al., 2014). The H5-ELP PBs were approximately $800 \mathrm{~nm}$ in diameter whereas the H5-hydrophobin PBs were substantially smaller, with an average diameter of $250 \mathrm{~nm}$. The H5-zein PBs reported herein were larger, with a diameter of 1-2 $\mu \mathrm{m}$, which is similar to the average size of endogenous zein bodies found in maize endosperm (Lending and Larkins, 1989). In contrast to the H5-ELP and H5-hydrophobin PBs, H5zein formed high density structures that were insoluble in nonreducing buffers, whereas H5-ELP and H5-hydrophobin fusion proteins could be extracted in $50 \mathrm{mM}$ Tris, $\mathrm{pH} 8.0$ (Phan et al., 2014). 
The H5-zein bodies described herein were used as a delivery vehicle for a model vaccine antigen. IgG responses were elicited in all mice immunized with H5-zein but HI assays indicated the absence of neutralizing antibodies. Our results agree with previous parenteral immunization studies using monomeric hemagglutinin fused to ELP, which, in contrast to trimeric hemagglutinin, also did not induce neutralizing antibodies (Phan et al., 2013). Although the formation of PBs involves multiple cross-linking via intermolecular disulfide bonds, this type of multimerization may not be sufficient to support the specific oligomerization state that appears to be required for the formation of specific native epitopes that may confer a seroprotective immune response. Proper trimerization may be required to complete the folding of hemagglutinin monomers and to induce conformational effects necessary for full antigenicity and the induction of neutralizing antibodies (Magadan et al., 2013). The introduction of a trimerization signal in addition to the assembly sequence may therefore be beneficial, as reported for H5-ELP fusions (Phan et al., 2013).

One remarkable outcome of our experiments was that a comparable immune response was elicited in all mice despite the H5-zein concentration being 100 times lower than the concentration of soluble $\mathrm{H} 5$ in the control group, which was administered with a strong adjuvant. Interestingly, the administration of an adjuvant together with the H5-zein bodies did not promote a stronger immune response, suggesting that the addition of the zein portion itself mediates an adjuvant effect. This agrees with Whitehead et al. (Whitehead et al., 2014), who recently reported that the immunogenicity of a recombinant antigen was increased in the presence of Zera ${ }^{\circledR}$, an assembly sequence that is very similar to the $\mathrm{N}$-terminal part of $\gamma$-zein (Torrent et al., 2009b), and the immunogenicity of the fusion protein could not be enhanced further by the inclusion of Freund's adjuvant. Similarly, the injection of synthetic zein microspheres that were loaded with ovalbumin resulted in higher IgG responses than the 'free' soluble protein (Hurtado-Lopez and Murdan, 2006a). This strongly supports the hypothesis that the zein $\mathrm{N}$-terminal portion possesses intrinsic adjuvant activity, although we cannot exclude the possibility that the observed adjuvant effect was mediated by another component of the PBs. Joseph et al. reported that zein-induced PBs isolated from leaves contain additional proteins that are trapped during biogenesis (Joseph et al., 2012).

An adjuvant effect conferred by a polymer-forming protein domain is not unexpected, given its similarity to the strategy of attaching a carrier protein such as albumin or keyhole limpet hemocyanin to antigens with poor immunogenicity (Harris and Markl, 1999). By definition, an adjuvant is characterized by its ability to enhance the immunogenic efficacy of antigens in the same formulation. This can be achieved by increasing the halflife of an antigen, improving antigen delivery to its effector sites, or providing immunostimulatory signals to enhance the immune response. The observed adjuvant effect of zein particles may reflect one or more of several relevant properties. First, hydrophobic synthetic block copolymers have been shown to confer stronger adjuvant properties than hydrophilic polymers (Newman et al., 1998; Hunter, 2002). Accordingly, the N-terminal part of $\gamma$-zein is partially hydrophobic, favoring intermolecular and membrane interactions (Kogan et al., 2002). It has also been reported that particulate antigens are transported more efficiently to murine splenic follicular dendritic cells in vivo in the absence of prior immunity, making them more immunogenic than soluble antigens (Link et al., 2012). This may also be reflected by the superior immunogenicity of hemagglutinin-containing virus-like particles compared to soluble hemagglutinin, even in the absence of an adjuvant (Shoji et al., 2015). Also, repetitive antigen display, structural, or molecular mimicry of the virus, particle-size dependent tissue penetration and trafficking to lymphatics and Toll-like receptor activation are possible mechanisms. In repetitive antigen display the spatial organization of the antigens on the particle surface facilitates B-cell receptor (antibody) co-aggregation, triggering and activation. This can support the production of longlived high-affinity neutralizing antibodies (Smith et al., 2013). Plant-derived PBs might also provide a specific spatial antigen organization favoring a successful repetitive antigen display. Alternatively, increased half life and stability of the zein fusions in the serum in vivo might be responsible for the enhanced immune response. The half-life of the antigen is likely to be extended due to encapsulation in the protein body. Indeed, pharmaceutical preparations encapsulated in zein particles in vitro remained in the blood for at least $24 \mathrm{~h}$ following intravenous delivery (Lai and Guo, 2011). Interestingly, the fusion of hemagglutinin to ELP repeats did not seem to increase immunogenicity although the propensity to form protein aggregates was confirmed in planta (Phan et al., 2013, 2014).

Zein has several favorable general characteristics as an adjuvant, i.e., it is stable at ambient temperatures and yet it is biodegradable, encouraging its use as a biopolymer for the coating and encapsulation of recombinant proteins such as erythropoietin (Bernstein et al., 1993). However, the potential immunogenic properties of zein must be taken into account (Hurtado-Lopez and Murdan, 2006a; Whitehead et al., 2014). We detected an immune response directed against $\gamma$-zein although the response was much weaker than that directed against $\mathrm{H} 5$, and when compared to the control group administered with $\mathrm{H} 5$ alone, the difference between the groups was not statistically significant. However, $30 \%$ of the mice injected with H5-zein showed an immune response above background levels. A significant immune response against the zein-like sequence Zera has been reported by (Whitehead et al., 2014), warranting further studies to assess the suitability of zein bodies as drug delivery vehicles for parenteral administration. Animal studies involving the injection of zeincoated erythropoietin (Bernstein et al., 1993) and ivermicin (Gong et al., 2011) did not indicate any adverse effects. Other storage proteins, including the wheat storage protein gliadin, have also been used as coatings to prepare various proteins and pharmaceuticals in vitro. GliSODin ${ }^{\circledR}$ for example is an oral treatment for oxidative stress, in which superoxide dismutase (SOD) is coated with gliadin (Cloarec et al., 2007). Although gliadin protects SOD from digestion, this storage protein is also linked to the autoimmune disorder celiac disease 
(Chaptal, 1957). Even so, this product has received market approval for human use.

\section{CONCLUSION}

Zein and similar plant storage proteins have long been investigated as carriers for pharmaceuticals including recombinant proteins. The direct encapsulation of pharmaceutical proteins in the production host is a simple approach that is less expensive than the production of synthetic microparticles. Our case study using a model vaccine antigen indicates that zein-induced PBs can be used as vaccine delivery vehicles that benefit from a value-added adjuvant effect, whereas the intrinsic immunogenicity of the zein component is low. The insertion of a trimerization signal fused to $\mathrm{H} 5$ will be tested to determine whether this leads to the assembly of structures that can elicit neutralizing antibodies against H5. It will also be of value to develop the in planta protein body encapsulation strategy for the production and delivery

\section{REFERENCES}

Ahmed, O. A., Hosny, K. M., Al-Sawahli, M. M., and Fahmy, U. A. (2015). Optimization of caseinate-coated simvastatin-zein nanoparticles: improved bioavailability and modified release characteristics. Drug Des. Dev. Ther. 9, 655-662. doi: 10.2147/DDDT.S76194

Bernstein, H., Mathiowitz, E., Morrel, E., Brickner, A., and Pabst, P. (1993). Erythropoietin Drug Delivery System (WO 1993025221).

Chaptal, J. (1957). Celiac disease caused by intolerance to gliadin from wheat, oats \& milk products. Pädiatrie 12, 737-747.

Cloarec, M., Caillard, P., Provost, J. C., Dever, J. M., Elbeze, Y., and Zamaria, N. (2007). GliSODin, a vegetal sod with gliadin, as preventative agent vs. atherosclerosis, as confirmed with carotid ultrasound-B imaging. Eur. Ann. Allergy Clin. Immunol. 39, 45-50.

Conley, A. J., Joensuu, J. J., Menassa, R., and Brandle, J. E. (2009). Induction of protein body formation in plant leaves by elastin-like polypeptide fusions. BMC Biol. 7:48. doi: 10.1186/1741-7007-7-48

Daamen, W. F., Veerkamp, J. H., Van Hest, J. C., and Van Kuppevelt, T. H. (2007). Elastin as a biomaterial for tissue engineering. Biomaterials 28, 4378-4398. doi: 10.1016/j.biomaterials.2007.06.025

de Virgilio, M., De Marchis, F., Bellucci, M., Mainieri, D., Rossi, M., Benvenuto, E., et al. (2008). The human immunodeficiency virus antigen Nef forms protein bodies in leaves of transgenic tobacco when fused to zeolin. J. Exp. Bot. 59, 2815-2829. doi: 10.1093/jxb/ern143

Floss, D. M., Sack, M., Stadlmann, J., Rademacher, T., Scheller, J., Stoger, E., et al. (2008). Biochemical and functional characterization of anti-HIV antibody-ELP fusion proteins from transgenic plants. Plant Biotechnol. J. 6, 379-391. doi: 10.1111/j.1467-7652.2008.00326.x

Geli, M. I., Torrent, M., and Ludevid, D. (1994). Two structural domains mediate two sequential events in [gamma]-zein targeting: protein endoplasmic reticulum retention and protein body formation. Plant Cell 6, 1911-1922. doi: 10.1105/tpc.6.12.1911

Gong, S. J., Sun, S. X., Sun, Q. S., Wang, J. Y., Liu, X. M., and Liu, G. Y. (2011). Tablets based on compressed zein microspheres for sustained oral administration: design, pharmacokinetics, and clinical study. J. Biomater. Appl. 26, 195-208. doi: 10.1177/0885328210363504

Gutierrez, S. P., Saberianfar, R., Kohalmi, S. E., and Menassa, R. (2013). Protein body formation in stable transgenic tobacco expressing elastin-like polypeptide and hydrophobin fusion proteins. BMC Biotechnol. 13:40. doi: 10.1186/14726750-13-40

Harris, J. R., and Markl, J. (1999). Keyhole limpet hemocyanin (KLH): a biomedical review. Micron 30, 597-623. doi: 10.1016/S0968-4328(99)00036-0 of further antigens, including candidates intended for oral application.

\section{AUTHOR CONTRIBUTIONS}

AH designed and carried out experiments, analyzed data, and wrote the manuscript. SM designed and carried out experiments and analyzed data. MT and EA carried out experiments, analyzed data, and contributed to the manuscript. HP, UG, and JL carried out experiments and analyzed data. UC and ES designed the study, analyzed data, and wrote the manuscript.

\section{ACKNOWLEDGMENTS}

The authors would like to acknowledge financial support by the Austrian Science Fund FWF (W1224 and I1461-B16). We are greatful to Dr. Jutta Veits, Friedrich-Loeffler-Institut, GreifswaldInsel Riems for providing the inactivated virus.

Hofbauer, A., Peters, J., Arcalis, E., Rademacher, T., Lampel, J., Eudes, F., et al. (2014). The induction of recombinant protein bodies in different subcellular compartments reveals a cryptic plastid-targeting signal in the $27-\mathrm{kDa}$ gammazein sequence. Front. Bioeng. Biotechnol. 2:67. doi: 10.3389/fbioe.2014.00067

Hofbauer, A., and Stoger, E. (2013). Subcellular accumulation and modification of pharmaceutical proteins in different plant tissues. Curr. Pharm. Des. 19, 5495-5502. doi: 10.2174/1381612811319310005

Hulse, D. J., Webster, R. G., Russell, R. J., and Perez, D. R. (2004). Molecular determinants within the surface proteins involved in the pathogenicity of $\mathrm{H} 5 \mathrm{~N} 1$ influenza viruses in chickens. J. Virol. 78, 9954-9964. doi: 10.1128/JVI.78.18.9954-9964.2004

Hunter, R. L. (2002). Overview of vaccine adjuvants: present and future. Vaccine 20(Suppl. 3), S7-S12. doi: 10.1016/S0264-410X(02)00164-0

Hurtado-Lopez, P., and Murdan, S. (2006a). An investigation into the adjuvanticity and immunogenicity of zein microspheres being researched as drug and vaccine carriers. J. Pharm. Pharmacol. 58, 769-774. doi: 10.1211/jpp.58.6.0007

Hurtado-Lopez, P., and Murdan, S. (2006b). Zein microspheres as drug/antigen carriers: a study of their degradation and erosion, in the presence and absence of enzymes. J. Microencapsul. 23, 303-314. doi: 10.1080/02652040500444149

Joseph, M., Ludevid, M. D., Torrent, M., Rofidal, V., Tauzin, M., Rossignol, M., et al. (2012). Proteomic characterisation of endoplasmic reticulum-derived protein bodies in tobacco leaves. BMC Plant Biol. 12:36. doi: 10.1186/14712229-12-36

Khan, I., Twyman, R. M., Arcalis, E., and Stoger, E. (2012). Using storage organelles for the accumulation and encapsulation of recombinant proteins. Biotechnol. J. 7, 1099-1108. doi: 10.1002/biot.201100089

Klenk, H. D., and Garten, W. (1994). Host cell proteases controlling virus pathogenicity. Trends Microbiol. 2, 39-43. doi: 10.1016/0966-842X(94)9 0123-6

Kogan, M. J., Dalcol, I., Gorostiza, P., Lopez-Iglesias, C., Pons, R., Pons, M., et al. (2002). Supramolecular properties of the proline-rich gamma-Zein N-terminal domain. Biophys. J. 83, 1194-1204. doi: 10.1016/S0006-3495(02) 75243-0

Lai, L. F., and Guo, H. X. (2011). Preparation of new 5-fluorouracil-loaded zein nanoparticles for liver targeting. Int. J. Pharm. 404, 317-323. doi: 10.1016/j.ijpharm.2010.11.025

Lammel, A. S., Hu, X., Park, S. H., Kaplan, D. L., and Scheibel, T. R. (2010). Controlling silk fibroin particle features for drug delivery. Biomaterials 31, 4583-4591. doi: 10.1016/j.biomaterials.2010.02.024

Lau, E. T., Giddings, S. J., Mohammed, S. G., Dubois, P., Johnson, S. K., Stanley, R. A., et al. (2013). Encapsulation of hydrocortisone and mesalazine in zein microparticles. Pharmaceutics 5, 277-293. doi: 10.3390/pharmaceutics5020277 
Lending, C. R., and Larkins, B. A. (1989). Changes in the zein composition of protein bodies during maize endosperm development. Plant Cell 1, 1011-1023. doi: $10.2307 / 3869002$

Lin, T., Lu, C., Zhu, L., and Lu, T. (2011). The biodegradation of zein in vitro and in vivo and its application in implants. AAPS PharmSciTech 12, 172-176. doi: 10.1208/s12249-010-9565-y

Link, A., Zabel, F., Schnetzler, Y., Titz, A., Brombacher, F., and Bachmann, M. F. (2012). Innate immunity mediates follicular transport of particulate but not soluble protein antigen. J. Immunol. 188, 3724-3733. doi: 10.4049/jimmunol.1103312

Liu, X., Sun, Q., Wang, H., Zhang, L., and Wang, J. Y. (2005). Microspheres of corn protein, zein, for an ivermectin drug delivery system. Biomaterials 26, 109-115. doi: 10.1016/j.biomaterials.2004.02.013

Llop-Tous, I., Madurga, S., Giralt, E., Marzabal, P., Torrent, M., and Ludevid, M. D. (2010). Relevant elements of a maize gamma-zein domain involved in protein body biogenesis. J. Biol. Chem. 285, 35633-35644. doi: 10.1074/jbc.M110.116285

Llop-Tous, I., Ortiz, M., Torrent, M., and Ludevid, M. D. (2011). The expression of a xylanase targeted to ER-protein bodies provides a simple strategy to produce active insoluble enzyme polymers in tobacco plants. PLOS ONE 6:e19474. doi: 10.1371/journal.pone.0019474

Lohcharoenkal, W., Wang, L., Chen, Y. C., and Rojanasakul, Y. (2014). Protein nanoparticles as drug delivery carriers for cancer therapy. Biomed Res. Int. 2014, 180549. doi: $10.1155 / 2014 / 180549$

Magadan, J. G., Khurana, S., Das, S. R., Frank, G. M., Stevens, J., Golding, H., et al. (2013). Influenza A virus hemagglutinin trimerization completes monomer folding and antigenicity. J. Virol. 87, 9742-9753. doi: 10.1128/JVI.00 471-13

Mainieri, D., Rossi, M., Archinti, M., Bellucci, M., De Marchis, F., Vavassori, S., et al. (2004). Zeolin. A new recombinant storage protein constructed using maize $\gamma$-zein and bean phaseolin. Plant Physiol. 136, 3447-3456.

Newman, M. J., Todd, C. W., and Balusubramanian, M. (1998). Design and development of adjuvant-active nonionic block copolymers. J. Pharm. Sci. 87, 1357-1362. doi: 10.1021/js980072c

Nitta, S. K., and Numata, K. (2013). Biopolymer-based nanoparticles for drug/gene delivery and tissue engineering. Int. J. Mol. Sci. 14, 1629-1654. doi: 10.3390/ijms14011629

Panyam, J., and Labhasetwar, V. (2003). Biodegradable nanoparticles for drug and gene delivery to cells and tissue. Adv. Drug Deliv. Rev. 55, 329-347. doi: 10.1016/S0169-409X(02)00228-4

Petros, R. A., and DeSimone, J. M. (2010). Strategies in the design of nanoparticles for therapeutic applications. Nat. Rev. Drug Discov. 9, 615-627. doi: $10.1038 / \mathrm{nrd} 2591$

Phan, H. T., Hause, B., Hause, G., Arcalis, E., Stoger, E., Maresch, D., et al. (2014). Influence of elastin-like polypeptide and hydrophobin on recombinant hemagglutinin accumulations in transgenic tobacco plants. PLoS ONE 9:e99347. doi: 10.1371/journal.pone.0099347

Phan, H. T., Pohl, J., Floss, D. M., Rabenstein, F., Veits, J., Le, B. T., et al. (2013). ELPylated haemagglutinins produced in tobacco plants induce potentially neutralizing antibodies against $\mathrm{H} 5 \mathrm{~N} 1$ viruses in mice. Plant Biotechnol. J. 11, 582-593. doi: 10.1111/pbi.12049

Podaralla, S., Averineni, R., Alqahtani, M., and Perumal, O. (2012). Synthesis of novel biodegradable methoxy poly(ethylene glycol)-zein micelles for effective delivery of curcumin. Mol. Pharm. 9, 2778-2786. doi: 10.1021/ mp2006455

Podaralla, S., and Perumal, O. (2010). Preparation of zein nanoparticles by $\mathrm{pH}$ controlled nanoprecipitation. J. Biomed. Nanotechnol. 6, 312-317. doi: $10.1166 /$ jbn. 2010.1137

Ruszczak, Z., and Friess, W. (2003). Collagen as a carrier for on-site delivery of antibacterial drugs. Adv. Drug Deliv. Rev. 55, 1679-1698. doi: 10.1016/j.addr.2003.08.007

Rybicki, E. P., Hitzeroth, II, Meyers, A., Dus Santos, M. J., and Wigdorovitz, A. (2013). Developing country applications of molecular farming: case studies in South Africa and Argentina. Curr. Pharm. Des. 19, 5612-5621. doi: $10.2174 / 1381612811319310015$

Shewry, P. R., and Halford, N. G. (2002). Cereal seed storage proteins: structures, properties and role in grain utilization. J. Exp. Bot. 53, 947-958. doi: $10.1093 /$ jexbot $/ 53.370 .947$

Shigemitsu, T., Masumura, T., Morita, S., and Satoh, S. (2013). Accumulation of rice prolamin-GFP fusion proteins induces ER-derived protein bodies in transgenic rice calli. Plant Cell Rep. 32, 389-399. doi: 10.1007/s00299-012-1372

Shoji, Y., Prokhnevsky, A., Leffet, B., Vetter, N., Tottey, S., Satinover, S., et al. (2015). Immunogenicity of H1N1 influenza virus-like particles produced in Nicotiana benthamiana. Hum. Vaccin. Immunother. 11, 118-123. doi: 10.4161/hv.34365

Smith, D. M., Simon, J. K., and Baker, J. R. Jr. (2013). Applications of nanotechnology for immunology. Nat. Rev. Immunol. 13, 592-605. doi: $10.1038 /$ nri3488

Stoger, E., Fischer, R., Moloney, M., and Ma, J. K. (2014). Plant molecular pharming for the treatment of chronic and infectious diseases. Annu. Rev. Plant Biol. 65, 743-768. doi: 10.1146/annurev-arplant-050213-035850

Takaiwa, F., Wakasa, Y., Takagi, H., and Hiroi, T. (2015). Rice seed for delivery of vaccines to gut mucosal immune tissues. Plant Biotechnol. J. 13, 1041-1055. doi: $10.1111 /$ pbi. 12423

Torrent, M., Llompart, B., Lasserre-Ramassamy, S., Llop-Tous, I., Bastida, M., Marzabal, P., et al. (2009a). Eukaryotic protein production in designed storage organelles. BMC Biol. 7:5. doi: 10.1186/1741-7007-7-5

Torrent, M., Llop-Tous, I., and Ludevid, M. D. (2009b). Protein body induction: a new tool to produce and recover recombinant proteins in plants. Methods Mol. Biol. 483, 193-208. doi: 10.1007/978-1-59745-407-0_11

Vaquero, C., Sack, M., Chandler, J., Drossard, J., Schuster, F., Monecke, M., et al. (1999). Transient expression of a tumor-specific single-chain fragment and a chimeric antibody in tobacco leaves. Proc. Natl. Acad. Sci. U.S.A. 96, 11128-11133. doi: 10.1073/pnas.96.20.11128

Wang, R., Tian, Z., and Chen, L. (2011). Nano-encapsulations liberated from barley protein microparticles for oral delivery of bioactive compounds. Int. J. Pharm. 406, 153-162. doi: 10.1016/j.ijpharm.2010.12.039

Whitehead, M., Ohlschläger, P., Almajhdi, F. N., Alloza, L., Marzábal, P., Meyers, A. E., et al. (2014). Human papillomavirus (HPV) type 16 E7 protein bodies cause tumour regression in mice. BMC Cancer 14:367. doi: 10.1186/1471-240714-367

Wiley, D. C., Skehel, J. J., and Waterfield, M. (1977). Evidence from studies with a cross-linking reagent that the haemagglutinin of influenza virus is a trimer. Virology 79, 446-448. doi: 10.1016/0042-6822(77)90371-3

Wilson, I. A., Skehel, J. J., and Wiley, D. C. (1981). Structure of the haemagglutinin membrane glycoprotein of influenza virus at 3 A resolution. Nature 289, 366-373. doi: 10.1038/289366a0

Zhong, Q., and Jin, M. (2009). Nanoscalar structures of spray-dried zein microcapsules and in vitro release kinetics of the encapsulated lysozyme as affected by formulations. J. Agric. Food Chem. 57, 3886-3894. doi: $10.1021 /$ jf803951a

Zou, T., and Gu, L. (2013). TPGS emulsified zein nanoparticles enhanced oral bioavailability of daidzin: in vitro characteristics and in vivo performance. Mol. Pharm. 10, 2062-2070. doi: 10.1021/mp400086n

Conflict of Interest Statement: The authors declare that the research was conducted in the absence of any commercial or financial relationships that could be construed as a potential conflict of interest.

Copyright (C) 2016 Hofbauer, Melnik, Tschofen, Arcalis, Phan, Gresch, Lampel, Conrad and Stoger. This is an open-access article distributed under the terms of the Creative Commons Attribution License (CC BY). The use, distribution or reproduction in other forums is permitted, provided the original author(s) or licensor are credited and that the original publication in this journal is cited, in accordance with accepted academic practice. No use, distribution or reproduction is permitted which does not comply with these terms. 